\title{
Caracterización de pacientes postoperados de cirugías electivas que ingresan a la Unidad de Cuidados Intensivos del Hospital de Clínicas
}

\author{
Walter Delgado (iD), Raúl Alonso (iD), Christian Sala (iD), Evanhy Vega \\ Universidad Nacional de Asunción, Facultad de Ciencias Médicas, Hospital de Clínicas, \\ Departamento Central de Anestesiología. San Lorenzo, Paraguay
}

Cómo referenciar este artículo/ How to reference this article:

\begin{abstract}
Delgado W, Alonso $R$, Sala $C$, Vega $E$. Caracterización de pacientes postoperados de cirugías electivas que ingresan a la Unidad de Cuidados Intensivos del Hospital de Clínicas. Mem. Inst. Investig. Cienc. Salud. 2021; 19(1): 86-91
\end{abstract}

\begin{abstract}
RE S U M E N
El ingreso a la Unidad de Cuidados Intensivos (UCI) de un paciente post operado es para optimizar el estado clínico corrigiendo trastornos relacionados con el estrés quirúrgico y las complicaciones intraoperatorias. El objetivo del estudio fue caracterizar los pacientes post operados que ingresaron a la UCI. Se realizó un estudio observacional, descriptivo, transversal en 163 pacientes post operados de cirugías electivas que ingresaron a UCI. La muestra representó el $4,6 \%$ de las cirugías electivas. El $94,5 \%$ fueron ingresos previstos y el 5,5\% imprevistos. Dentro de los casos previstos $(n=154)$, el $68 \%$ ingresaron estables y extubados para monitoreo, el $19 \%$ por inestabilidad hemodinámica y el $13 \%$ por imposibilidad de extubación. El promedio de días de internación en la UCI fue $4 \pm 3$ días. 7 pacientes fallecieron $(4,3 \%)$. En conclusión, se encontró una alta frecuencia de ingresos previstos. La mayoría fueron para un monitoreo postoperatorio por la envergadura de la cirugía o por las comorbilidades del paciente. La mayoría de los pacientes ingresaron hemodinámicamente estables y extubados. Los servicios que más ingresos requirieron fueron Cirugía General y Neurocirugía. El tiempo promedio de internación fue 4 días. La mortalidad fue $4,3 \%$.
\end{abstract}

Palabras clave: Cuidados intensivos, procedimientos quirúrgicos operativos, mortalidad.

\section{Characterization of elective surgery post-operative patients admitted to the Intensive Care Unit of the Hospital de Clínicas}

\begin{abstract}
The admission to the Intensive Care Unit (ICU) of a post-operative patient is to optimize clinical status by correcting disorders related to surgical stress and intraoperative complications. The objective of the study was to characterize the postoperated patients who entered to the ICU. An observational, descriptive, crosssectional study was conducted in 163 post-operative patients of elective surgeries that entered ICU. The sample represented the $4.6 \%$ of elective surgeries, $94.5 \%$ was planned admissions and 5.5\% unexpected. Within the expected cases ( $n-154), 68 \%$ entered stable and extubated for monitoring, $19 \%$ for hemodynamic instability and $13 \%$ for impossibility of extubation. The average number of days of ICU internment were $4 \pm 3$ days and seven patients died (4,3\%). In conclusion, a high frequency of expected admissions was found. Most of them were for postoperative monitoring by the size of the surgery or by the patient's comorbidity. Most patients entered hemodynamically stable and extubated. The services that required most admittances Fecha de recepción: Noviembre 2020. Fecha de aceptación: Marzo 2021

*Autor correspondiente: Walter Delgado. Universidad Nacional de Asunción, Facultad de Ciencias Médicas, Hospital de Clínicas, Departamento Central de Anestesiología. San Lorenzo, Paraguay Email: walterdelga@gmail.com
\end{abstract}


were General Surgery and Neurosurgery. The average hospitalization time were 4 days and mortality was $4.3 \%$.

Keywords: Intensive care, operational surgical procedures, mortality.

\section{INTRODUCCIÓN}

El éxito operatorio depende de la interacción entre el acto quirúrgico realizado, la salud previa del paciente y los eventos intraoperatorios y posoperatorios ${ }^{(1)}$. El riesgo de eventos adversos perioperatorios está condicionado por la enfermedad del paciente antes de la cirugía, la prevalencia de comorbilidades y la urgencia de la operación, así como de la magnitud, el tipo y la duración del procedimiento quirúrgico ${ }^{(2)}$.

El ingreso post operatorio a UCI se ha considerado como un procedimiento estándar en ciertos tipos de cirugía electivas de alto riesgo, mientras que en otros casos puede darse de forma imprevista cuando ocurre alguna complicación en el intraoperatorio.

El objetivo del ingreso en UCI de un paciente post operado es para optimizar el estado clínico corrigiendo los trastornos inmediatos relacionados con el estrés quirúrgico ${ }^{(3,4)}$ y las complicaciones intraoperatorias (hipotermia, desequilibrios del medio interno, edemas, anemia).

Pero a su vez es también importante recalcar que a veces el ingreso a la UCI puede tener efectos secundarios: como un mayor riesgo de infecciones nosocomiales, un menor acceso a la movilización temprana, la sobredosificación de la sedación, la falta de sueño, el estrés y el delirio(5).

El objetivo de este estudio fue caracterizar los pacientes post operados que ingresan a la UCI describiendo los motivos, el tiempo de estadía, las especialidades quirúrgicas y mortalidad.

Esta caracterización de la ocupación de camas en UCI por pacientes post operados es muy relevante para cualquier centro quirúrgico para poder planificar las necesidades según el tipo de cirugía y la gravedad de los pacientes quirúrgicos.

\section{MATERIALES Y MÉTODOS}

Se realizó un estudio observacional, descriptivo, de corte transversal con un marco temporal retrospectivo en pacientes post operados de cirugías electivas en el Pabellón Quirúrgico Central del Hospital de Clínicas que ingresaron a la Unidad de Cuidados Intensivos en el periodo de julio de 2018 a julio de 2019.

Se incluyeron a pacientes de ambos sexos, de todas las especialidades quirúrgicas que ingresaron a UCI en el postoperatorio inmediato. Se excluyeron a pacientes pediátricos, pacientes postoperados que fueron derivados a UCI desde la sala de internación y las cirugías de urgencias.

El muestreo fue no probabilístico, de casos consecutivos y se realizó una revisión de fichas médicas en el archivo central de la UCI.

Las variables analizadas fueron: edad, tiempo de estadía, sexo, causas de ingreso, especialidad quirúrgica, previsión del ingreso, óbito. Las mismas fueron registradas en una base de datos creada en Excel 2016. Los resultados fueron analizados con estadística descriptiva.

Se respetaron los principios éticos y se mantuvo la confidencialidad de los datos de los pacientes. No se presentaron conflictos de intereses.

\section{RESULTADOS}

Se estudiaron 163 pacientes que ingresaron a UCI en el postoperatorio inmediato. Estos pacientes representaron el $4,6 \%$ de las cirugías electivas $(n=3.489)$.

Las distribución por género de los pacientes en UCI $(n=163)$ fue similar, $52 \%$ pacientes masculinos y $48 \%$ femeninos. La edad media fue $51 \pm 15$ años, con un rango de 19 a 87 años.

La distribución de pacientes según las especialidades quirúrgicas se detalla en la Tabla 1. 
Tabla 1: Distribución de pacientes según las especialidades quirúrgicas. Post operatorio inmediato de cirugías electivas. UCI. Hospital de Clínicas. Julio 2018 - julio 2019. $n=163$

\begin{tabular}{lllll}
\hline Especialidad & $\begin{array}{l}\text { Ingresos } \\
\text { previstos }\end{array}$ & $\begin{array}{l}\text { Ingresos } \\
\text { imprevistos }\end{array}$ & $\begin{array}{l}\text { Total de } \\
\text { pacientes }\end{array}$ & $\begin{array}{l}\text { Frecuencia } \\
\text { total }\end{array}$ \\
\hline Cirugía general & 49 & 2 & 51 & $31,3 \%$ \\
Neurocirugía & 48 & 0 & 48 & $29,5 \%$ \\
Urología & 26 & 5 & 31 & $19 \%$ \\
Cirugía torácica & 15 & 0 & 15 & $9,2 \%$ \\
Cirugía cardíaca & 12 & 0 & 12 & $7,3 \%$ \\
Otorrinolaringología & 3 & 1 & 4 & $2,5 \%$ \\
Mastología & 0 & 1 & 1 & $0,6 \%$ \\
Traumatología & 1 & 0 & 1 & $0,6 \%$ \\
\hline Total & 154 & 9 & 163 & $100 \%$ \\
\hline
\end{tabular}

El número de ingresos previstos fue $154(94,5 \%)$ y los ingresos imprevistos fueron $9(5,5 \%)$.

Dentro de los casos previstos $(n=154)$, el $68 \%$ de los pacientes ingresaron estables y extubados para un monitoreo postoperatorio $y / 0$ para estabilizar desequilibrios intraoperatorios del medio interno teniendo en cuenta la envergadura de la cirugía o las comorbilidades de los pacientes. El 19\% ingresaron con una inestabilidad hemodinámica y el $13 \%$ con asistencia mecánica respiratoria por imposibilidad de extubación en el postoperatorio inmediato.

Dentro de los casos imprevistos $(n=9)$, la inestabilidad hemodinámica fue la causa principal $(77,7 \%)$ de ingreso a UCI. En el resto de los casos $(22,22 \%)$ fue la imposibilidad de extubación por alteraciones respiratorias.

El promedio de días de internación en la UCI fue $4 \pm 3$ días.

Del total de pacientes, 156 fueron dados de alta de la UCI y pasaron a sala internación y 7 pacientes obitaron, evidenciando una mortalidad de 4,3\%.

\section{DISCUSIón}

La admisión a la UCI puede disminuir la morbilidad y la mortalidad asociadas con enfermedades graves o cirugía de alto riesgo, pero también da como resultado un mayor costo y potencialmente una mayor duración de estancia hospitalaria ${ }^{(6,7)}$.

Varios estudios refieren que la proporción de pacientes que en el posoperatorio inmediato ingresan a UCI oscilan entre el $2 \%$ y el $4,5 \%{ }^{(8-10)}$. Pero esta frecuencia puede variar en los diversos centros hospitalarios teniendo en cuenta las variables sociodemográficas, las especialidades quirúrgicas y los protocolos respectivos ${ }^{(8,9)}$. En nuestro estudio se evidenció una proporción ligeramente mayor $(4,6 \%)$. Se debe tener en cuenta que existen muchos criterios para decidir la necesidad de cuidados posoperatorio en la UCI. Uno de ellos tiene que ver con la propia cirugía en el cual la duración de la misma está muy relacionada a desequilibrios del medio interno que pueden necesitar una corrección y un monitoreo posterior. Es importante reconocer que en nuestro Hospital Escuela los médicos residentes realizan la mayor parte de las cirugías tutoreados por Especialistas. Dentro de una curva de aprendizaje se utiliza un mayor tiempo quirúrgico que muchas veces puede favorecer desequilibrios del medio interno que predispongan la necesidad de un cuidado intensivo post operatorio.

Por otra parte, se evidenció que la mayor proporción de pacientes que ingresaron a la UCI lo hicieron en condiciones estables. Esto indica que en muchas situaciones previstas no existieron grandes alteraciones fisiopatológicas que ameriten cuidados complejos. Considerando esta situación, la proporción de pacientes que en el posoperatorio van a la UCI podría disminuirse con el gerenciamiento adecuado de una Unidad de Cuidados Post Anestésicos en la cual puedan realizarse cuidados intermedios y los pacientes puedan tener estancias cortas para correcciones y 
monitoreo. Esto beneficiaría a cualquier centro hospitalario teniendo en cuenta que las camas de UCI constituyen generalmente un recurso limitado.

En los países desarrollados, los pacientes sometidos a cirugía no cardíaca de alto riesgo constituyen una parte importante de los ingresos a la UCI ${ }^{(11,12)}$. Encontramos que los pacientes que ingresaron posterior a una cirugía cardíaca constituyeron el $7.3 \%$ de los casos, por lo que la mayor proporción de pacientes ingresados fueron pacientes sometidos a cirugías electivas no cardíacas, quienes ameritaron su ingreso ya sea que por la envergadura de la cirugía o por su estado clínico.

Un hallazgo llamativo en nuestro estudio fue la cantidad de pacientes que ingresaron a la UCI en forma imprevista que representaron el 5.5\%, una frecuencia superior a algunos reportes que indicaron frecuencias menores al $1 \%{ }^{(9)}$. En este contexto es interesante mencionar que muchos de los casos pueden ameritar solo una estabilización o compensación del paciente durante estancias cortas en Unidades de Cuidados Intermedios o Unidades de Cuidados Post Anestésicos (UCPA) debidamente equipados para el efecto. Por lo tanto, la optimización de la UCPA con personal capacitado y equipamiento adecuado podrían disminuir la necesidad de ingresar a algunos pacientes a UCI.

Copeland et al. ${ }^{(13,14)}$ desarrollaron un sistema de puntuación para proporcionar un análisis tanto retrospectivo como prospectivo del riesgo de morbilidad y mortalidad posquirúrgicas. El Score POSSUM es el sistema de puntuación predictiva más ampliamente validado que se utiliza en la atención perioperatoria. La correcta aplicación del mismo ayuda a predecir la necesidad de cuidados intensivos en el pos operatorio y disminuir imprevisibilidad de dicha situación. Aunque muchas veces es difícil predecir que paciente va a tener una complicación intra operatoria, la correlación entre el tipo de cirugía y las comorbilidades del paciente ayudan a realizar una planificación pre operatoria. En nuestro estudio hemos evidenciado una proporción menor al 5\% de pacientes que han ingresado a la UCI en forma imprevista lo que es un porcentaje aceptable en el contexto de la planificación preoperatoria de una cirugía electiva.

La mayor frecuencia de ingresos se dieron en pacientes de la Especialidad de cirugía general y neurocirugía, hecho coincidente con algunas publicaciones ${ }^{(15)}$. Debemos considerar que esto puede ser variable dependiendo de las cirugías que se realizan con más frecuencia en los centros quirúrgicos. En el Hospital de Clínicas, la mayor cantidad de cirugías electivas constituyen las realizadas por los cirujanos generales. Por ende, esta mayor casuística genera directamente una mayor demanda de camas en la UCI por esta Especialidad.

No debemos confundir esto con la proporción de pacientes que requieren UCI estratificados por Especialidad ya que si nos referimos a este contexto, recalcamos que todas las cirugías cardíacas y la mayoría de las neurocirugías necesitan pasar a UCI en el postoperatorio. Algunos estudios ${ }^{(15,16)}$ refieren que la mayor causa de ingreso en el post operatorio inmediato son las alteraciones hemodinámicas. La mayor proporción de pacientes, dentro de los ingresos previstos, ingresaron extubados y estables para correcciones mínimas del medio interno y monitoreo por la envergadura quirúrgica de la cirugía lo que constituye un buen indicador del manejo intraoperatorio.

Los ingresos imprevistos se produjeron principalmente por complicaciones intraoperatorias relacionadas a hemorragias, lo que favoreció una frecuencia elevada de inestabilidad hemodinámica en este grupo de pacientes.

El tiempo promedio de estadía fue de cuatro días, tiempo similar a lo referido en otras publicaciones ${ }^{(16)}$. El delineamiento de estrategias para disminuir el tiempo de permanencia en las UCI es muy importante a fin de disminuir las complicaciones inherentes a la estadía en la misma.

En este estudio se evidenció una mortalidad un poco superior a lo referido por algunos autores $(4.3 \% \text { vs. } 2.3 \%)^{(7,15)}$. La mayor mortalidad perioperatoria generalmente está relacionada a situaciones imprevistas del intraoperatorio y a las comorbilidades mayores de los pacientes por lo que es muy importante una 
planificación adecuada del plan anestésico y quirúrgico en el contexto clínico del paciente.

Los pacientes ingresados a UCI en el postoperatorio inmediato representaron el $4,6 \%$ de las cirugías electivas. La mayoría de los ingresos fueron previstos en el preoperatorio $(94,5 \%)$. La mayoría de los pacientes ingresaron hemodinámicamente estables y extubados. Los servicios que más ingresos requirieron fueron Cirugía General y Neurocirugía. El tiempo en promedio de internación fue de 4 días. Se encontró una mortalidad del 4,3\%.

\section{REFERENCIAS BIBIOGRÁFICAS}

1. Sobol JB, Wunsch H. Triage of high-risk surgical patients for intensive care. Critical care [Internet]. 2011; 15: 227 [citado 2019 Jul 27]. Disponible en: http://ccforum.com/content/15/2/217

2. Delgado MCM, Vidal FG. Perioperative intensive care medicine. Med Intensiva Delgado MCM, Vidal FG. Perioperative intensive care medicine. Med Intensiva (English Ed [Internet]. 2019; (xx). Available from: 10.1016/j.medine.2019.03.010

3. Clarke A, Murdoch $H$, Thomas MJ, Cook TM, Peden CJ: Mortality and postoperative care after emergency laparotomy. European Journal of Anaesthesiology (EJA). 2011, 28 (1): 16-19.

10.1097/EJA.0b013e32833f5389.

4. Bui JQ, Mendis RL, van Gelder JM, Sheridan MM, Wright KM, Jaeger $M$. Is postoperative intensive care unit admission a prerequisite for elective craniotomy? J Neurosurg. 2011 Dec; 115(6): 1236-41.

doi:

10.3171/2011.8.JNS11105. Epub 2011 Sep 2. PMID: 21888476.

5. Taccone P, Langer T, Grasselli G. Do we really need postoperative ICU management after elective surgery? Intensive Care Med. [Internet]. 2017; 43(7):1037-1038. [citado 2019 Jul 27]. Disponible en: 10.1007/s00134-0174814-0

6. Dueñas-Castell C, Ortiz Ruiz G. La atención médica en la unidad de cuidado intensivo en Colombia. Acta Colomb Cuid Intens. 2014; 14(1):5-7.

7. Pérez Gutiérrez, Norton, Análisis de mortalidad de pacientes en Unidad de Cuidados Intensivos en un Hospital del Departamento del Meta, Colombia. Investigaciones Andina [Internet]. 2016; 18(33):1605-1624. Recuperado de:

https://www.redalyc.org/articulo.oa?id= 239053104002

8. Pearse RM, Moreno RP, Bauer P, Pelosi $P$, Metnitz $P$, Spies $C$, et al; European Surgical Outcomes Study (EuSOS) group for the Trials groups of the European Society of Intensive Care
Medicine and the European Society of Anesthesiology. Mortality after surgery in Europe: a 7-day cohort study. Lancet. 2012; 380: 1059-1065.

9. Meziane $M$, Sidi $E$, Abdelghafour $M$, Bensghir B, Hicham A, Redouane $A$, et al. Unplanned Intensive Care Unit Admission following Elective Surgical Adverse Events: Incidence, Patient Characteristics, Preventability, and Outcome. Indian Journal of Critical Care Medicine 2017.21. 10.4103/ijccm.IJCCM_428_16.

Disponible

en: https://www.researchgate.net/publicati on/314391435_Unplanned_Intensive_C are_Unit_Admission_following_Elective_ Surgical_Adverse_Events_Incidence_Pat ient_Characteristics_Preventability_and _Outcome

10. Satyawan B, Shinde V, Chaudhari L. Audit of intensive care unit admissions from operating room. Indian J Anaesth. 2006; 50: 193-200.

11. Ogawa $T$, Inoue $S$, Inada $M$, Kawaguchi M. Postoperative intensive care unit admission does not affect outcomes in elective surgical patients with severe comorbidity. Med Intensiva 2020; 44 (4):

216-225. 10.1016/j.medin.2019.01.003.

12. Harrison DA, Lone NI, Haddow $\mathrm{C}$, MacGillivray $M$, Khan $A$, Cook $B$, et al. External validation of the Intensive Care National Audit \& Research Centre (ICNARC) risk prediction model in critical care units in Scotland. BMC Anesthesiol. 2014; 14:116.

13. Copeland GP, Jones D, Walters M. POSSUM: a scoring system for surgical audit. $\mathrm{Br}$ J Surg. $1991 ; 78355-360$

14. Copeland GP. The POSSUM System of Surgical Audit. Arch Surg. 2002; 137(1):15-19.

doi: 10.1001/archsurg.137.1.15

15. Uzman S, Yilmaz $Y$, Toptas M, Akkoc I, Gul Y, H, Toptas $Y$. A retrospective analysis of postoperative patients admitted to the intensive care unit. Hippokratia. 2016 Jan-Mar; 20(1): 3843. 
16. Prieto J, Astroña C, Noe Quiroz P. Frecuencia de complicaciones y tiempo de estadía hospitalaria en cirugía cardiovascular, Hospital Caja Petrolera Santa Cruz - 2014. Rev Cient Cienc Méd [Internet].

2015 [citado 2020 Sep 29]; 18(1):27-

30

Disponible en: http://www.scielo.org.bo/scielo.php?scri pt $=$ sci_arttext\&pid $=$ S1817$74332015000100006 \&$ Ing $=$ es.
17. De Cassia R, Rejane E, Moraes M, Azzolin K. Gravedad de pacientes de cirugía cardíaca: un análisis evolutivo según el TISS-28. Rev Latino-Am Enfermagem 2010; 18(1): 07 pantallas. Disponible en: http://www.scielo.br/scielo.php?pid $=$ S0104-

$11692010000100010 \&$ script $=$ sci_arttex t\&tlng $=\mathrm{es}$ 